\title{
Modelo para alocação de instrumentos com previsão de redundância de medição em sistemas com medições es- cassas.
}

\author{
M. V. A. NARCISO ${ }^{1}$, E. C. DO VALLE ${ }^{1}$ e R. A. KALID ${ }^{1}$ \\ ${ }^{1}$ Universidade Federal da Bahia, Departamento de Engenharia Química \\ Email para contato: marcosnarciso@ufba.br
}

\begin{abstract}
RESUMO - Alocar novos medidores de vazão de água em uma planta industrial é um desafio, devido a estas serem escassas e, especialmente, pela demanda crescente no uso racional deste recurso. As soluções apresentadas para este tipo de problema, baseadas no conceito de Qualidade da Informação (QI) preveem a instalação de um único medidor por ponto. O objetivo deste trabalho é apresentar uma extensão do modelo padrão de alocação de instrumentos que inclua a redundância de hardware, ou seja, a instalação de dois ou mais sensores em um mesmo ponto de instalação. Com esta modificação o modelo de alocação está apto a lidar em sistemas de transferência de custódia, os quais são tipicamente redundantes.
\end{abstract}

\section{INTRODUÇÃO}

Em plantas químicas já consolidadas, o processo de escolha de um novo instrumento é normalmente definido, ou pela necessidade de uma nova malha de controle, ou na necessidade de se conhecer uma nova variável. Ou seja, critérios técnicos como a observabilidade do sistema de medição ou a necessidade da redundância da informação para uma melhoria na controlabilidade do sistema não são utilizados no processo decisório.

Em sistemas de alto valor agregado como, por exemplo, o insumo principal em uma planta química diversos métodos foram desenvolvidos. Václavek e Loučka (1976) foram os primeiros a explorar este tipo de problema com o uso da teoria de grafos para a garantia da observabilidade do sistema. Madron e Veverka (1992) foram os primeiros a utilizar um modelo de otimização matemática através de duas funções-objetivo: custo e precisão máxima. Bagajewicz (1997) propôs a utilização de uma programação não linear inteiro mista (MINLP), onde há o uso de uma variável binária que indica a situação de um medidor candidato à instalação. Bagajewicz e Sánchez (2000b) generalizaram o modelo anteriormente proposto para casos de realocação de sensores pré-existentes e atualização do sistema de medição.

Estes métodos funcionam bem para sistemas de alto valor agregado, pois possuem um número suficientes de medições instaladas. Em sistemas de medição de água este cenário não é encontrado. Normalmente só há medição na entrada ou na saída da unidade em estudo, ou em ambas, sem o detalhamento necessário para um amplo conhecimento da topologia da utilidade. Narciso et al. (2012) propôs um modelo de alocação de instrumentos a partir do conceito da Qualidade da Informação (QI) (Martins et al., 2010) e da otimização do tipo MINLP (Bagajewicz e Sánchez, 2000b) para sistemas com medições escassas. A QI tem origem na estatística bayesiana e é obtido através das informações 
coletadas sobre a variável como, por exemplo, medições diretas e indiretas, experiências de campo, simulações de processo e documentação existente. A QI pode ser definida por Martins et al. (2010):

$$
Q I_{i} \propto \frac{y_{i}}{\sigma^{2}} \rightarrow Q I_{i}=\kappa \cdot \frac{y_{i}}{\sigma^{2}}
$$

em que $\kappa$ é uma constante de proporcionalidade, $y_{i}$ é o valor da variável reconciliada e $\sigma_{i}^{2}$ a variância de $y_{i}$.

Porém, este método funciona bem para sistemas sem redundância de hardware, ou seja, sistemas onde só é permitido a instalação de um único instrumento por ponto. É conhecido que existem sistemas de medição de água com alto valor agregado como, por exemplo, os circuitos de vapor ou as linhas de água desmineralizada, os quais requerem um maior conhecimento de suas vazões. Estes sistemas requerem redundância de hardware, principalmente quando envolvem a transferência de custódia. Neste trabalho é apresentada uma proposta de extensão do modelo MINLP proposto por Narciso et al. (2012) visando a utilização deste para a adição de redundância de hardware. O modelo será apresentado e discutido ao longo deste trabalho, sucedido com a resolução de um exemplo encontrado na literatura com a devida análise dos seus resultados.

\section{FORMULAÇÃO DO MODELO}

Narciso et al. (2011) propuseram um modelo de otimização para alocação de instrumentos em sistemas com medições escassas, o qual é definido por:

$$
\min _{q_{i}} \sum_{j \in M_{p}} a_{j} \cdot\left(\frac{y_{i}}{\widehat{Q I}_{j}(\mathbf{q})}\right)^{2}
$$

sujeito a

$$
\begin{array}{cc}
\sum_{i \in M_{l}} c_{i} \cdot q_{i} \leq c_{T} & \forall i \in M_{l} \\
q_{i}=\{0,1\} & \forall i \in M_{l}
\end{array}
$$

em que $a_{j}$ é um peso, $y_{i}$ é a variável reconciliada, $\widehat{Q I}_{j}(q)$ é a QI após a reconciliação referente à variável $j$ de interesse ao sistema de medição, $c_{i}$ é o custo individual da medição em $i, c_{T}$ é o investimento total disponível e q é o vetor das variáveis binárias $q_{i}$ que indicam a presença de medição.

Porém, se há mais de um instrumento candidato disponível para a medição $i$ a funçãoobjetivo da Equação 2 precisa ser alterada com a adição de uma nova variável binária e a adição de novas restrições. Assumindo $n_{i}^{m}$ o número de medidores candidatos disponíveis para o ponto de medição $i, c_{i, k}\left(k=1, \ldots, n_{i}^{m}\right)$ o custo associado da medição em $i$ para cada alternativa disponível e que para cada variável $i$ haja uma variável binária $p_{i, k}(k=$ $\left.1, \ldots, n_{i}^{m}\right)$ a qual indica qual alternativa será instalada no ponto $i$, em que:

$$
p_{i, k}= \begin{cases}1 & \text { caso o medidor } k \text { seja instalado em } i \\ 0 & \text { caso não seja instalado. }\end{cases}
$$


A função investimento total disponível, presente nas restrições do sistema, passará a depender de $p$, sendo descrita por (Bagajewicz, 1997):

$$
C(p)=\sum_{i \in M l} \sum_{k=1}^{n_{i}^{m}} c_{i, k} \cdot p_{i, k} .
$$

Portanto a função-objetivo é reescrita por:

$$
\min _{q_{i}} \sum_{j \in M_{p}} a_{j} \cdot\left(\frac{y_{i}}{\widehat{Q I}_{j}(\mathbf{q})}\right)^{2}
$$

sujeito a

$$
\begin{aligned}
\sum_{i \in M l} \sum_{k=1}^{n_{i}^{m}} c_{i, k} \cdot p_{i, k} \leq c_{T} & \forall i \in M_{l} \\
\sum_{k=1}^{n_{i}^{m}} p_{i, k} \leq 1 & \forall i \in M_{l} \\
p_{i, k}=\{0,1\} & \forall i \in M_{l}, \forall k=1, \ldots, n_{i}^{m} .
\end{aligned}
$$

Neste formato a função-objetivo da Equação 5 garante a instalação de somente um medidor candidato $k$ em cada ponto de medição $i$. Porém, alterando-se a segunda restrição para:

$$
\sum_{k=1}^{n_{i}^{m}} p_{i, k} \leq m_{i}
$$

garante que até $m_{i}$ instrumentos redundantes sejam instalados por ponto de medição.

Quando $m_{i}>1$, a nova Qualidade de Informação do ponto de medição $\left(\widehat{Q I}_{j}(\mathbf{q})\right)$ pode ser facilmente obtida através da combinação das QIs individuais dos medidores.

\section{EXEMPLO}

\subsection{Processo Simplificado}

Figura 1 - Processo simplificado. Fonte: (Bagajewicz, 1997)

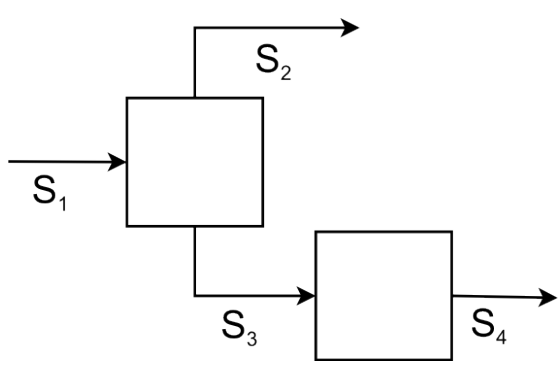


Considere o processo simplificado, representado na Figura 1, cujo modelo foi apresentado originalmente por Bagajewicz (1997). O processo contém 4 correntes, todas sem medições pré-existentes, e 2 nós. Os dados de vazão deste sistema estão na segunda coluna da Tabela 1.

Tabela 1 - Dados para a resolução do exemplo.

\begin{tabular}{l|rrrrr} 
& Vazão $/ t \cdot h^{-1}$ & $\widehat{Q I}^{*}(\mathbf{q}) /$ adim. & $Q I_{3 \%} /$ adim. & $Q I_{2 \%} /$ adim. & $Q I_{1 \%} /$ adim. \\
\hline$y_{1}$ & 150,1 & - & 3,3 & 5,0 & 10,0 \\
$y_{2}$ & 52,3 & 6,7 & 3,3 & 5,0 & 10,0 \\
$y_{3}$ & 97,8 & - & 3,3 & 5,0 & 10,0 \\
$y_{4}$ & 97,8 & 5,0 & 3,3 & 5,0 & 10,0
\end{tabular}

Assumindo que para cada ponto candidato a receber uma medição há disponível medidores de 3\%, $2 \%$ e 1\%, com um custo individual de US\$ 800,00, US\$ 1 500,00 e US\$ 2 500,00 respectivamente. Os equivalente em QI dos valores das precisões dos instrumentos candidatos estão na quarta, quinta e sexta colunas da Tabela 1.

Bagajewicz (1997) propôs que as correntes 1 e 4 são as de interesses ao processo, sendo que as precisões requeridas são de $1,5 \%$ e 2,0\%, os quais tem sua representação em QI na terceira coluna da Tabela 1. Com um investimento total de US\$ 3 000,00 duas soluções foram obtidas pelos autores e estas foram reproduzidas pelo modelo da Equação 5 (ver soluções \#1 e \#2 na Tabela 2), porém sem redundância.

Para a necessidade de uma redundância, ou seja $m_{i}=2$, e com um investimento total de US\$ 3 100,00 duas novas soluções foram encontradas. Estas soluções também foram encontradas pelo modelo da Equação 5 e estão detalhadas nas soluções \#3 e \#4 da Tabela 2.

Tabela 2 - Resultados para a alocação ótima sem redundância (casos \#1 e \#2) e com redundância (casos \#3 e \#4).

\begin{tabular}{crrrr}
\hline Solução & $y_{1}$ & $y_{2}$ & $y_{3}$ & $y_{4}$ \\
\hline$\# 1$ & - & $2 \%$ & $2 \%$ & - \\
$\# 2$ & - & $2 \%$ & - & $2 \%$ \\
\hline$\# 3$ & $3 \%$ & $3 \%$ & $2 \%$ & - \\
$\# 4$ & $3 \%$ & $3 \%$ & - & $2 \%$ \\
\hline
\end{tabular}

\section{CONCLUSÕES}

A análise comparativa do exemplo comprovou que a metodologia proposta é uma alternativa viável para realizar a alocação de instrumentos em sistemas industriais com poucas, ou até mesmo, ausência de medições.

A possibilidade de definir a necessidade ou não de redundância de hardware torna o modelo versátil e aplicável a sistemas de alta confiabilidade, como os de transferência de custódia, assim como a aplicação em projetos de instrumentação, onde são definidos pontos de medição. 


\section{REFERÊNCIAS}

\section{Referências}

Ali, Y.; NARAsimhan, S. Sensor network design for maximizing reliability of linear processes. AIChE Journal, 39(5), 820-828, 1993.

Bagajewicz, M.; SÁnchez, M. Cost-optimal design of reliable sensor networks. Computers $\& 6$ Chemical Engineering, 23(11-12), 1757-1762, 2000a.

Bagajewicz, M.; SÁnChez, M. Reallocation and upgrade of instrumentation in process plants. Computers \& Chemical Engineering, 24(8), 1945-1959, 2000 b.

BAGAJewicz, M. J. Design and retrofit of sensor networks in process plants. AIChE Journal, 43(9), 2300-2306, 1997.

Madron, F.; Veverka, V. Optimal selection of measuring points in complex plants by linear models. AIChE Journal, 38(2), 227-236, 1992.

Martins, M. A.; Amaro, C. A.; Souza, L. S.; Kalid, R. A.; Kiperstok, A. New objective function for data reconciliation in water balance from industrial processes. Journal of Cleaner Production, 18(12), 1184-1189, 2010.

Narciso, M.; Valle, E.; Braga, E.; Kiperstok, A.; Kalid, R.; Alegre, P. A MINLP formulation to optimize sensor allocation using reconciled data in systems with fewer measurements. Em Congresso Brasileiro de Metrologia, p. 1-5, Natal, 2011.

Narciso, M. V. A.; Valle, E.; Kiperstok, A.; Kalid, R. D. A. VAlidaÇÃO DE MODELO MINLP PARA ALOCAÇÃO ÓTIMA DE INSTRUMENTOS UTILIZANDO DADOS RECONCILIADOS EM SISTEMAS SEM MEDIÇÕES. Em Secchi, A., editor, COBEQ 2012, p. 1-9, Buzios, 2012.

VÁClaVeK, V.; LouČKA, M. Selection of measurements necessary to achieve multicomponent mass balances in chemical plant. Chemical Engineering Science, 31(12), 1199-1205, 1976. 